\title{
Boolean like algebras
}

\author{
Antonio Ledda ${ }^{1}$, Tomasz Kowalski ${ }^{1}$, Francesco Paoli ${ }^{1}$, and Antonino Salibra ${ }^{3}$ \\ 1 Università di Cagliari, Cagliari, Italia \\ antonio.ledda@unica.it, paoli@unica.it \\ 2 La Trobe University, Melbourne, Bundoora, Australia \\ T.Kowalski@latrobe.edu. au \\ 3 Università Ca'Foscari, Venezia, Italia \\ salibra@dsi.unive.it
}

\begin{abstract}
Using Vaggione's concept of central element in a double pointed algebra, we introduce the notion of Boolean like variety as a generalisation of Boolean algebras to an arbitrary similarity type. Appropriately relaxing the requirement that every element be central in any member of the variety, we obtain the more general class of semi-Boolean like varieties, which still retain many of the pleasing properties of Boolean algebras. We prove that a double pointed variety is discriminator iff it is semi-Boolean like, idempotent, and 0-regular. This theorem yields a new Maltsev-style characterisation of double pointed discriminator varieties. Moreover, we show that every idempotent semi-Boolean-like variety is term equivalent to a variety of noncommutative Boolean algebras with additional regular operations.
\end{abstract}

\section{The results}

Boolean algebras have an exceptionally rich and smooth structure theory, of which Stone's representation theorem is a prominent example. What is so special about Boolean algebras that is responsible for this nice behaviour? Given a similarity type $\nu$, can we always find a class of algebras of type $\nu$ that displays Boolean-like features? And what does it mean, for an algebra of a given type $\nu$ that may not exhibit such desirable properties, to have at least a subset of Boolean elements that behave well? To address these questions, we use the concept, due to Vaggione [20, of a central element in a double pointed algebra, meaning an element which induces therein, in a specified sense, a pair of complementary factor congruences. Roughly speaking, given a similarity type $\nu$ including at least two constants but otherwise fully arbitrary, we associate the presence of a "well-behaved Boolean core" in a $\nu$-algebra with the presence of a retract of central elements, and we identify Boolean $\nu$-algebras with $\nu$-algebras where every element is central. In order to fully appreciate what properties of Boolean algebras are responsible for the most important results concerning this variety, however, the issue is best addressed in a step-by-step fashion. Therefore, following [13], we will decompose the property of centrality into several equational properties, trying to investigate what happens when some of them are satisfied but other ones are dropped. This approach will give rise to a few successive approximations to a full-fledged notion of "Boolean algebra of arbitrary similarity type".

Our work ties nicely with at least three research streams that have received considerable attention in universal algebra and in the investigation into the mathematical foundations of computer science:

- (Weak) Boolean product representations. It has been known for a long time that Stone's representation theorem, perhaps the most distinctive result characterising Boolean algebras (or Boolean rings), can be generalised to a much larger class of algebras. The appropriate tool to attain this goal is the technique of Boolean products, which can be loosened to the notion of weak Boolean product to take care of somewhat less manageable cases (see 
e.g. 9]). Pierce [17 proved that every commutative ring with unit is representable as a weak Boolean product of directly indecomposable rings; Stone's representation theorem follows as a corollary by observing that (i) every congruence is a factor congruence in a Boolean ring; (ii) the 2-element ring of truth values is the unique directly indecomposable Boolean ring. The technique of Boolean products underwent remarkable developments over the subsequent years (see e.g. [5, Ch. 4.8]), giving rise to further generalisations of Stone's theorem by Comer (covering the case of algebras with Boolean factor congruences 6]) and Vaggione [20.

- Discriminator varieties and noncommutative lattice theory. Discriminator varieties 21] are referred to by Burris and Sankappanavar as

the most successful generalization of Boolean algebras to date, successful because we obtain Boolean product representations (which can be used to provide a deep insight into algebraic and logical properties) [5, p. 186].

One of the most elegant characterisations of discriminator varieties in the pointed case was obtained by Bignall and Leech [2, who linked them to a noncommutative generalisation of Boolean algebras called left handed skew Boolean $\cap$-algebras. More precisely, Bignall and Leech proved that: (i) the variety of type $(3,0)$ generated by the class of all pointed discriminator algebras $\mathbf{A}=(A ; t, 0)$, where $t$ is the discriminator function on $A$ and 0 is a constant, is term equivalent to the variety of left handed skew Boolean $\cap$-algebras; (ii) every pointed discriminator variety is term equivalent to a variety of left handed skew Boolean $\cap$-algebras with additional compatible operations. This result can be easily adapted to the double pointed case 1 , which is particularly significant in that the variety of Boolean algebras is double pointed [3. Some more steps in this direction are taken in what follows.

- Algebraic investigation of the if-then-else construct. There is a thriving literature on abstract treatments of the fundamental if-then-else construct of computer science, starting with McCarthy's seminal investigations [16. On the algebraic side, one of the most influential approaches originated with a paper by Bergman [1]. Bergman modelled the if-then-else by considering Boolean algebras acting on sets: if the Boolean algebra of actions is the 2-element algebra, one simply sets $1(a, b)=a$ and $0(a, b)=b$ (see e.g. 8 for details). Alternative perspectives make recourse to dynamic algebras [18] or Kleene algebras with tests [10]. The approach followed in this paper originates with Dicker's axiomatisation of Boolean algebras in the type $(3,0,0)$ [7, and differs from Bergman's in that the if-then-else is treated as a proper algebraic ternary operation $q$ on a double pointed algebra $\mathbf{A}$, having the property that for every $a, b \in A, q(1, a, b)=a$ and $q(0, a, b)=b$. The resulting variety of Church algebras is investigated in [12, 13, 14, 15] and is one of the fundamental notions in the present work as well.

In greater detail, our presentation is structured as follows. First, we will dispatch some necessary preliminaries, and we recall from [13] the definition of Church algebra, introducing the concept of central element and providing an equational characterisation thereof. In a generic Church algebra, of course, there is no need for the set of central elements to comprise all of the algebra. Church algebras where this is the case are (unimaginatively) called Boolean like, while the ones that miss the mark by possibly failing one designated equation (in other words, where every element is semi-central) are termed semi-Boolean like. We provide a characterisation of

\footnotetext{
${ }^{1}$ Following [3], we say that a class of similar algebras is double pointed if its type has at least two constants that realise distinct elements in any nontrivial member of the class.
} 
Boolean like varieties as discriminator varieties in which the directly indecomposable members are two-element algebras. Moreover, we prove several properties of the pure semi-Boolean like variety, e.g. that it has no congruence identities. Then, we give a purely algebraic characterisation of semi-Boolean like varieties along the lines of the one provided in 3 for discriminator varieties. Afterwards, we use the previous concepts and results to provide several descriptions of double pointed discriminator varieties. With such an aim in mind, we consider semi-Boolean like algebras where a term definable meet-like binary operation is idempotent. We prove that a double pointed variety is discriminator iff it is semi-Boolean like, idempotent, and 0-regular. This theorem yields a new Maltsev-style characterisation of double pointed discriminator varieties.

In every Church algebra $\mathbf{A}$ central elements are the universe of a Boolean algebra that is isomorphic to the Boolean algebra of complementary factor congruences of $\mathbf{A}$ [19, Theorem 3.7], which means that every central element induces a direct decomposition of $\mathbf{A}$. Here, we prove that the factors in this decomposition can be described by exploiting a generalisation of the relativisation construction for Boolean algebras. Then, we proceed to deal with the stronger notions of semi-Boolean-like algebras and varieties. The new results we present are essentially two: we point out the exact relationship between semi-Boolean-like varieties and the quasi-discriminator varieties of [11, and we provide semi-Boolean-like algebras with an explicit weak Boolean product representation with directly indecomposable factors. Finally, idempotent semi-Boolean-like algebras are investigated. We consider a noncommutative generalisation of Boolean algebras and prove - along the lines of similar results available for pointed discriminator varieties [2] or for varieties with a commutative ternary deduction term [4 - that every idempotent semi-Boolean-like variety is term equivalent to a variety of noncommutative Boolean algebras with additional operations.

\section{References}

[1] Bergman G.M., "Actions of Boolean rings on sets", Algebra Universalis, 28, 1991, pp. 153-187.

[2] Bignall R.J., Leech J., "Skew Boolean algebras and discriminator varieties", Algebra Universalis, 33, 1995, pp. 387-398.

[3] Blok W.J., Pigozzi D., "On the structure of varieties with equationally definable principal congruences III", Algebra Universalis, 32, 1994, pp. 545-608.

[4] Blok W.J., Pigozzi D., "On the structure of varieties with equationally definable principal congruences IV", Algebra Universalis, 31, 1994, pp. 1-35.

[5] Burris S.N., Sankappanavar H.P., A Course in Universal Algebra, Springer, Berlin, 1981.

[6] Comer S., "Representations by algebras of sections over Boolean spaces", Pacific Journal of Mathematics, 38, 1971, pp. 29-38.

[7] Dicker R.M., "A set of independent axioms for Boolean algebra", Proceedings of the London Mathematical Society, 3, 13, 1963, pp. 20-30.

[8] Jackson M., Stokes T., "Semigroups with if-then-else and halting programs", International Journal of Algebra and Computation, 19, 7, 2009, pp. 937-961.

[9] Jónsson B., "Congruence distributive varieties", Mathematica Japonica, 42, 1995, pp. 353-402.

[10] Kozen D.C., "On Hoare Logic and Kleene algebra with Tests", ACM Transactions on Computational Logic, 1, 2000, pp. 60-76.

[11] Ledda A., Paoli F., Spinks M., Kowalski T. "Quasi-subtractive varieties 2", submitted.

[12] Manzonetto G., Salibra A., "Boolean algebras for lambda calculus", Proceedings of the 21th IEEE Symposium on Logic in Computer Science (LICS 2006), IEEE Computer Society, 2006, pp. 317-326 
[13] Manzonetto G., Salibra A., "From $\lambda$-calculus to universal algebra and back", in $M F C S$ '08, volume 5162 of LNCS, 2008, pp. 479-490.

[14] Manzonetto G., Salibra A., "Applying universal algebra to lambda calculus", Journal of Logic and Computation, 20, 4, 2010, pp. 877-915.

[15] Manzonetto G., Salibra A., "Lattices of equational theories as Church algebras", in C. Drossos et al., Proceedings of the Seventh Panhellenic Logic Symposium, Patras University Press, 2009, pp. 117-121.

[16] McCarthy J., "A basis for a mathematical theory of computation", in P. Braffort and D. Hirschberg (Eds.), Computer Programming and Formal Systems, North-Holland, Amsterdam, 1963, pp. 33-70.

[17] Peirce R.S., Modules over Commutative Regular Rings, Memoirs of the American Mathematical Society, 1967.

[18] Pratt V.R., Dynamic Algebras: Examples, Constructions, Applications, Technical Report MIT/LCS/TM-138, M.I.T. Laboratory for Computer Science, July 1979.

[19] Salibra A., Ledda A., Paoli F., Kowalski T., "Boolean-like algebras", Algebra Universalis, 69, 2, pp. 113-138.

[20] Vaggione D., "Varieties in which the Pierce stalks are directly indecomposable", Journal of Algebra, 184, 1996, pp. 424-434.

[21] Werner H., Discriminator Algebras, Studien zur Algebra und ihre Anwendungen, Band 6, Akademie-Verlag, Berlin, 1978. 